\title{
Adult Onset Cerebral X-Linked Adrenoleuokodystrophy in 18 Cases
}

\author{
Yuko Furuhashi ${ }^{*}$, Masaya Ishikawa ${ }^{2}$ \\ ${ }^{1}$ Section of Health Care Center, Shizuoka University, Shizuoka, Japan \\ ${ }^{2}$ Saginuma Kouen Clinic, Kawasaki, Japan

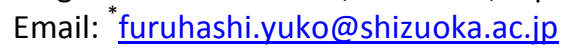

Received 30 April 2015; accepted 13 June 2015; published 17 June 2015

Copyright (C) 2015 by authors and Scientific Research Publishing Inc.

This work is licensed under the Creative Commons Attribution International License (CC BY).

http://creativecommons.org/licenses/by/4.0/

(c) (i) Open Access

\begin{abstract}
Adrenoleukodystrophy (ALD) is an X-linked inherited metabolic disease associated with the accumulation of very long chain fatty acids (VLCFA) in the nervous system, adrenal cortex, and testes. At least seven phenotypes can be distinguished, which are Addison only, childhood, adolescent and adult cerebral ALD, adrenomyeloneuropathy (AMN), and symptomatic or asymptomatic carriers. Children most often develop rapidly a progressive cerebral disease, whereas adults rarely develop a cerebral disease. The majority of adult-onset ALD patients are AMN. The prognosis of ALD remains unpredictable in individual patients. Family history can be very informative. The plasma VLCFA assay permits precise diagnosis. Specific changes on brain Magnetic Resonance Imaging (MRI) can have diagnostic utility. However, there is considerable overlap among adult-onset leukodystrophies. Adult onset form of cerebral X-linked ALD (AOCALD) is a rare disease. The disease progresses rapidly with widespread demyelination of the cerebral hemispheres. AOCALD is an important differential diagnosis for adults with psychiatric symptoms and progressive cognitive changes. In this article, we review on case reports of AOCALD.
\end{abstract}

\section{Keywords}

Adrenoleukodystrophy, Adult Onset, Cerebrum, Hallucination, Delusion

\section{Introduction}

ALD is an X-linked inherited metabolic disease associated with very long chain fatty acids (VLCFA) accumulation in the nervous system, adrenal cortex, and testes [1] [2].

It is caused by a mutation in the ABCD1 gene on chromosome Xq28. A number of different mutations in the

*Corresponding author. 
ABCD1 gene have been reported to be associated with X-linked ALD [3]-[10]. The gene product is a peroxisomal membrane protein, which belongs to the family of peroxisomal membrane ABC half-transporter proteins. The genetic defect leads to decreased peroxisomal $\alpha$-oxidation of VLCFA and subsequent accumulation of VLCFA mainly in the central nervous system and adrenal glands as well as in plasma. Accumulation of VLCFA is associated with progressive central and peripheral demyelination, adrenocortical insufficiency, and hypogonadism. Its prevalence is currently estimated at 1 in 20,000 - 50,000 [11]. The clinical manifestations of ALD range widely. At least seven phenotypes can be distinguished, which are Addison only, childhood, adolescent and adult cerebral ALD, AMN with or without cerebral demyelination, and symptomatic or asymptomatic carriers [12]. Children most often develop rapidly a progressive cerebral disease, whereas adults rarely develop a cerebral disease. In the most severe phenotype, affected patients develop cerebral demyelination with inflammation which is rapidly progressive and usually fatal within 2 years after onset. This phenotype presents most frequently in early childhood (childhood cerebral ALD; CCALD), but can also occur in adolescence or adulthood. In seven phenotypes, two most frequent are childhood cerebral ALD (CCALD) and adrenomyeloneuropathy (AMN) [13]. Childhood cerebral ALD (CCALD) and AMN account for more than 75\% of all X-linked ALD [13].

The adrenomyeloneuropathy (AMN) phenotype usually develops between the 30 years and 50 years of life and is gradually progressive. AMN represents the core clinical symptoms in X-linked ALD. The neurologic disability is slowly progressive initially. The main symptoms of AMN are gait disturbances, adrenal insufficiency, bladder problems, as well as scarce scalp hair [14]. The neurological symptoms are related to a myeloneuropathy. Adrenocortical and testicular insufficiency can occur in isolation (Addison-only), but also in combination with any of the other phenotypes. There is no correlation between the duration or severity of endocrine dysfunction and the severity of the myeloneuropathy. The majority of adult-onset ALD patients are AMN. The adult onset form of cerebral X-linked ALD (AOCALD) is a rare disease and less than 3\% of X-linked patients present with ALD [13] [14]. The phenotypic presentation of AOCALD is mainly characterized by a gradual development of behavior changes and cognitive dysfunction [2]. The disease progresses rapidly with widespread demyelination of the cerebral hemispheres. Hallucinations and delusions are not common as initial symptoms, however, we reported the case of AOCALD heralded by auditory hallucinations and delusions [15]. Specific treatment should be effective at psychiatric stage before the occurrence of irreversible neurological lesions. General practitioners should bear in mind the importance of performing neurological and radiological examinations on psychiatric patients with chronic illnesses in order to diagnose correctly.

In this article, we present an overview of 18 case reports on AOCALD.

\section{Methods}

An electronic search of databases was conducted through academic search engine, MEDLINE.

Terms were entered for searching in the title and abstract of articles. The search set used the terms "Adrenoleykodystrophy (ALD)" or "adult onset" or "cerebrum” or "case report".

The search sets included all papers published from January 1995 to January 2015. The search was limited to journals written in English. The search produced 25 articles. All papers were then manually reviewed to ensure the studies investigated AOCALD.

\section{Results and Discussion}

\section{Over View of Reviewed Studies}

A total of 18 studies met the criteria for inclusion in this review.

The inclusion criteria were 1) diagnosis of probable X-linked ALD as confirmed by ABCD1 gene or serum VLCFA, 2) age of onset is older than 20 years old, 3) presence of cerebral demyelination confirmed by brain MRI or CT (cerebral ALD).

Table 1 provides an overview of the reviewed articles. The average age of cases was 33.4 years old (20 years old to 62 years old), and all cases were male. In these cases [3]-[10] [15]-[24], the symptomatology resembles childhood cerebral ALD (CCALD) such as progressive cognitive impairment and loss of memory. However, the early cognitive disturbance is rarely recognized by their families or at work in adults [4] [6] [7]. In 18 cases, progressive cognitive decline is common as initial symptoms [3]-[10] [15]-[24]. Three cases reported psychiatric symptoms as initial symptoms [3] [15] [21]. These are aggressivity and abulia [3], hallucinations and delusions 
Table 1. Summary of reviewed case studies.

\begin{tabular}{|c|c|c|c|}
\hline Author & Age of case & Initial symptoms & How to diagnosis \\
\hline Carra-Dalliere et al. [3] & 24 & Psychiatric disturbances, spatial disorientation, memory loss & $\begin{array}{l}\text { Family history, VLCFA, } \\
\text { MRI, ABCD } 1 \text { gene }\end{array}$ \\
\hline Brownstone E. et al. [16] & 50 & Disorientation, petulance, psychomotor agitation & VLCFA, MRI \\
\hline Inoue et al. [4] & 48 & Personality change, irritability & VLCFA, MRI, ABCD 1 gene \\
\hline Furuhashi Y. et al. [15] & 21 & Hallucination, delusion & VLCFA, MRI, brain biopsy \\
\hline Li et al. [5] & 37 & Progressive gait unsteadiness, impotence, incontinence & $\begin{array}{l}\text { family history, VLCFA, MRI, } \\
\text { ABCD } 1 \text { gene }\end{array}$ \\
\hline Dohle C.I. et al. [6] & 40 & Progressive cognitive decline, seizure & $\begin{array}{l}\text { VLCFA, MRI, ABCD } 1 \text { gene, } \\
\text { brain biopsy }\end{array}$ \\
\hline Saito T. et al. [7] & 62 & Dementia, spastic paraparesis & VLCFA, MRI, ABCD 1 gene \\
\hline Sutovsky S. et al. [8] & 37 & Progressive personality change & VLCFA, MRI, ABCD 1 gene \\
\hline Hitomi T. et al. [9] & 20 & Progressive hemiparesis, gait disturbance & VLCFA, MRI, ABCD 1 gene \\
\hline Larner A.J. [10] & 40 & Behavioral changes & VLCFA, MRI, ABCD 1 gene \\
\hline Luda E. et al. [17] & 37 & Memory disturbance, personality changes & VLCFA, MRI \\
\hline Sakakibara R. et al. [18] & 34 & Progressive spastic paraparesis, genitourinary dysfunction & VLCFA, MRI \\
\hline Farrell D.S. et al. [19] & 30 & Slurred speech, poor balance, headache, deteriorating vision & $\begin{array}{l}\text { family history, VLCFA, MRI, } \\
\text { ABCD } 1 \text { gene, }\end{array}$ \\
\hline Tan E.K. et al. [20] & 24 & Addison’s disease, slurred speech, memory disturbance & VLCFA, MRI \\
\hline Garside S. et al. [21] & 31 & Tardive dyskinesia, psychosis & VLCFA, MRI \\
\hline Ochi K. et al. [22] & 24 & Unstable gait, dysarthria, writing disturbance, finger tremor & VLCFA, MRI \\
\hline Leo R.J. [23] & 42 & Addison's disease, irritable, impulsive & VLCFA, MRI \\
\hline Waragai M. et al.[24] & 30 & Progressive cerebellar ataxia, spastic paraparesis & VLCFA, MRI \\
\hline
\end{tabular}

MRI, Magnetic resonance imaging; VLCFA, Very long chain fatty acids.

[15], and mania including impulsivity, loudness, and perseveration [21]. Some studies reported that the phenotypic presentation of AOCALD is mainly characterized by a gradual development of psychiatric symptoms mimicking schizophrenia or psychosis [1] [2] [12]. However, in 18 cases, there was a case that reported hallucinations and delusions appeared as initial symptoms [15]. Hallucinations and delusions were rare symptoms as initial symptoms.

All cases were performed brain MRI and plasma VLCFA levels. Specific changes on brain Magnetic Resonance Imaging (MRI) can have diagnostic utility.

A study suggested that over a period of ten years, approximately $20 \%$ of patients with AMN developed additional cerebral demyelination [25]. In 18 cases, several cases revealed that patients with AMN developed cerebral ALD [5] [7] [18] [22] [24]. As the study [25] and these cases, patients with AMN can develop cerebral demyelination. It is important to follow the patients with AMN or Addison's disease by re-examinations of MRI.

\section{Diagnosis}

The majority of adult-onset ALD patients is AMN. The incidence of AOCALD is less than 3\% [13] [14]. AOCALD should be considered in the differential diagnosis of leukoencephalopathy in an adult even though AOCALD is a rare disease. When ALD is suspected, the initial diagnostic step consists of analysis of VLCFA in plasma. Most laboratories measure the absolute concentration of C26:0 as well as the C24:0/C22:0 and C26:0/ C22:0 ratios [13]. However, it is difficult to diagnose patients with AOCALD correctly when psychiatric symptoms are initially isolated without more specific symptoms or a familial history [15]. Family history can be very informative [3] [5] [19]. All 18 cases were performed brain MRI [3]-[10] [15]-[24]. MRI can have diagnostic 
utility, however, there is considerable overlap among adult-onset leukodystrophies. Thus, the predictive value of even more sophisticated MRI techniques is limited and there is still an essential need for biological markers that can predict disease progress. Metabolic screening reveals increased VLCFA [3]-[10] [15]-[24]. The next step is to confirm the diagnosis by performing ABCD1 mutation analysis [3] [4] [6]-[10] [12] [19].

In adult males presenting with adrenocortical insufficiency, X-linked ALD should be considered and determination of plasma VLCFA levels should be performed [12]. Furthermore, adult males presenting cognitive and neurological symptoms with white matter lesions on brain MRI should be considered for X-linked ALD and determination of plasma VLCFA levels should be performed [12]. In adult men, the most common presenting symptom of X-linked ALD is a chronic myelopathy [12]. After ruling out a compressive myelopathy by MRI of the spinal cord and other common causes of chronic myelopathy, X-ALD should be considered [12]. In adult males with confluent white matter changes, X-ALD should be considered, especially when there is increased signal intensity on T2-weighed and FLAIR sequences in the parieto-occipital region and the splenium of the corpus callosum [3]-[10] [12] [15]-[24]. Metabolic screening reveals increased VLCFA, the next step is to confirm the diagnosis by performing ABCD1 mutation analysis [3] [4] [6]-[10] [12] [19].

\section{Treatment}

Oral administration of Lorenzo's oil normalizes or significantly lowers the levels of VLCFA in plasma [15]. However, the clinical efficacy of Lorenzo's oil and the clinical indications for use of Lorenzo's oil are controversial [12]. Approximately 70\% of AMN patients develop primary adorenocortical insufficiency before the onset of neurological symptoms [25]. The treatment of adorenocortical insufficiency in X-linked ALD is no different from in patients with primary adrenal insufficiency. Glucocorticoid replacement therapy dose not influence the development or progression of neurological symptoms [25].

Treatment is mainly symptomatic. If cerebral ALD is advanced at the time of diagnosis, prognosis is poor [6] [8] [10] [12] [15] [18] [23]. There is no proven treatment for AOCALD. There is currently no curative or preventive treatment for most of patients with AOCALD.

However, bone marrow transplantation (BMT) has been reported to have a long-term beneficial effect in childhood onset ALD early in the clinical course [9]. Despite significant mortality risk, allogeneic haematophietic cell transplantation (HCT) remains the only therapeutic intervention that can halt the progression of cerebral demyelination in X-ALD, provided the procedure is performed very early stage when affected boys or men have no or minor symptoms due to cerebral demyelinating disease [12].

Regular follow-up in AMN remains important. Some studies reported that 20\% of adult males with AMN phenotypes will develop AOCALD later in life [12] [25]. It is important to study if an allogeneic HCT can be successful. In addition, the challenge of the future is to discover risk factors for the development of cerebral ALD [12].

\section{Conclusions}

$\mathrm{X}$-linked ALD is an intriguing disorder that is still incompletely understood and limited treatment options.

In addition, the incidence of AOCALD is rare and AOCALD is sometimes diagnosed as other diseases. It is important to recognize X-linked ALD because treatment is available in some cases such as HCT in early stage of X-linked ALD. We realized that general practitioners should bear in mind the importance of performing neurological and radiological examinations on psychiatric patients with chronic illnesses in order to diagnose correctly. Increased awareness of this disorder will lead to accurate diagnosis, appropriate treatment selection, and genetic counseling.

\section{References}

[1] Luda, E. and Barisone, M.G. (2001) Adult-Onset Adrenoleukodystrophy. A Clinical and Neuropsychological Study. Neurological Science, 22, 21-25. http://dx.doi.org/10.1007/s100720170032

[2] Sarah, G., Patricia, I.R., Anthony, J.L. and Michael, F.M. (1999) Late-Onset Adrenoleukodystrophy Associated with Long-Standing Psychiatric Symptoms. Journal of Clinical Psychiatry, 60, 460-468. http://dx.doi.org/10.4088/JCP.v60n0708

[3] Carra-Dalliere, C., Scherer, C., Ayrignac, X., Menjot de Champfleur, N., Bellesme, C., Labauge, P. and Verny, C. (2013) Adult-Onset Cerebral X-Linked Adrenoleukodystrophy with Major Contrast-Enhancement Mimicking Ac- 
quired Disease. Clinical Neurology and Neurosurgery, 15, 1906-1907. http://dx.doi.org/10.1016/j.clineuro.2013.05.006

[4] Inoue, S., Terada, S., Matsumoto, T., Ujike, H. and Uchitomi, Y. (2012) A Case of Adult-Onset Adrenoleukodystrophy with Frontal Lobe Dysfunction: A Novel Point Mutation in the ABCD1 Gene. Internal Medicine, 51, 1403-1406. http://dx.doi.org/10.2169/internalmedicine.51.6899

[5] Li, J.Y., Hsu, C.C. and Tsai, C.R. (2010) Spinocerebellar Variant of Adrenoleukodystrophy with a Novel ABCD1 Gene Mutation. Journal of Neurological Sciences, 290, 163-165. http://dx.doi.org/10.1016/j.jns.2009.12.002

[6] Dohle, C.I., Bannykh, S.I., Hisama, F.M. and Baehring, J.M. (2009) Leukoencephalopathy in Adults: Is It Adrenoleukodystrophy? A Case Report and Molecular Analysis. Journal of Neurological Sciences, 285, 235-237. http://dx.doi.org/10.1016/j.jns.2009.06.012

[7] Saito, T., Mizuno, T., Watanabe, T., Ida, M. and Nakase, H. (2008) Rapid Exacerbation in an Elderly Case of AdultOnset X-Linked Adrenoleukodystrophy with Cerebral Corticospinal Tract Involvement. Archives of Neurology, 65, 416-417. http://dx.doi.org/10.1001/archneur.65.3.416

[8] Sutovský, S., Petrovic, R., Chandoga, J. and Turcáni, P. (2007) Adult Onset Cerebral form of X-Linked Adrenoleukodystrophy with Dementia of Frontal Lobe Type with New L160P Mutation in ABCD1 Gene. Journal of Neurological Sciences, 263, 149-153. http://dx.doi.org/10.1016/j.jns.2007.01.082

[9] Hitomi, T., Mezaki, T., Tomimoto, H., Ikeda, A., Shimohama, S., Okazaki, T., Uchiyama, T. and Shibasaki, H. (2005) Long-Term Effect of Bone Marrow Transplantation in Adult-Onset Adrenoleukodystrophy. European Journal of Neurology, 12, 807-810. http://dx.doi.org/10.1111/j.1468-1331.2005.01055.x

[10] Larner, A.J. (2003) Adult-Onset Dementia with Prominent Frontal Lobe Dysfunction in X-Linked Adrenoleukodystrophy with R152C Mutation in ABCD1 Gene. Journal of Neurology, 10, 1253-1254. http://dx.doi.org/10.1007/s00415-003-0172-7

[11] Van Geel, B.M., Assies, J., Vevering, G.J. and Barth, P.G. (1994) Predominance of Adrenomyeloneuropathy Phenotype of X-Linked Adrenoleukodystrophy in the Netherlands: A Survey of 30 Kindred. Neurology, 44, 2243-2246. http://dx.doi.org/10.1212/WNL.44.12.2343

[12] Engleen, M., Kemp, S., de visser, M., van Geel, B.M., Wandres, R.J., Auboung, P. and Poll-The, B.T. (2012) X-Linked Adrenoleukodystrophy (X-ALD): Clinical Presentation and Guideline for Diagnosis, Follow-Up and Management. Orphanet Journal of Rare Disease, 7, 51. http://dx.doi.org/10.1186/1750-1172-7-51

[13] Van Geel, B.M., Assies, J., Wanders, R.J.A. and Barth, P.G. (1997) X Linked Adrenoleukodystrophy: Clinical Presentation, Diagnosis, and Therapy. Journal of Neurology Neurosurgery \& Psychiatry, 63, 4-14. http://dx.doi.org/10.1136/jnnp.63.1.4

[14] Moster, H.W., Moster, A.B., Naidu, S. and Bregin, A. (1991) Clinical Aspects of Adrenoleukodystrophy and Adrenomyeloneuropathy. Development Neuroscience, 13, 254-264. http://dx.doi.org/10.1159/000112170

[15] Furuhashi, Y. and Ishikawa, M. (2011) Adult-Onset Adrenoleukodytrophy Heralded by Auditory Hallucinations and Delusions. Psychosomatics, 52, 492-493. http://dx.doi.org/10.1016/j.psym.2011.01.033

[16] Brownstone, E., Voigtländer, T., Baumhackl, U. and Finsterer, J. (2013) Epilepsy in Adult X-Linked Adrenoleucodystrophy Due to the Deletion c.1415-1416delAG in Exon 5 of the ABCD1-Gene. Gene, 513, 71-74. http://dx.doi.org/10.1016/j.gene.2012.10.076

[17] Luda, E. and Barisone, M.G. (2001) Adult-Onset Adrenoleukodystrophy: A Clinical and Neuropsychological Study. Neurological Sciences, 22, 21-25. http://dx.doi.org/10.1007/s100720170032

[18] Sakakibara, R., Fukutake, T., Arai, K., Katayama, K., Mori, M. and Hattori, T. (2001) Unilateral Caudate Head Lesion Stimulating Brain Tumour in X-Linked Adult Onset Adrenoleukodystrophy. Journal of Neurology Neurosurgery \& Psychiatry, 70, 414-415. http://dx.doi.org/10.1136/jnnp.70.3.414

[19] Farrell, D.S., Lawden, M.C. and Messios, N. (2000) Radiologically Selective Visual Pathway Involvement in Adult Onset Cerebral Adrenoleukodystrophy. Journal of Neurology Neurosurgery \& Psychiatry, 68, 249-251. http://dx.doi.org/10.1136/jnnp.68.2.249a

[20] Tan, E.K., Lim, S.H., Chan, L.L., Wong, M.C. and Tan, K.P. (1999) X-Linked Adrenoleukodystrophy: Spinocerebellar Variant. Clinical Neurology and Neurosurgery, 101, 137-140. http://dx.doi.org/10.1016/S0303-8467(99)00028-1

[21] Garside, S., Rosebush, P.I., Levinson, A.J. and Mazurek, M.F. (1999) Late-Onset Adrenoleukodystrophy Associated with Long-Standing Psychiatric Symptoms. Journal of Clinical Psychiatry, 60, 460-468. http://dx.doi.org/10.4088/JCP.v60n0708

[22] Ochi, K., Noda, K., Kawakami, H., Oka, M., Imon, Y., Mimori, Y. and Nakamura, S. (1998) Dentato-Rubral Tract Involvement in Adult-Onset Adrenoleukodystrophy. American Journal of Neuroradiology, 19, 1904.

[23] Leo, R.J. (1998) Behavioral Changes and Affective Instability Associated with Adult-Onset Adrenoleukodystrophy. Psychosomatics, 2, 176-177. http://dx.doi.org/10.1016/s0033-3182(98)71369-6 
[24] Waragai, M., Takaya, Y., Hayashi, M., Shibata, N. and Kobayashi, M. (1996) MRI of Adrenoleukodystrophy Involving Predominantly the Cerebellum and Brain Stem. Neuroradiology, 38, 788-791. http://dx.doi.org/10.1007/s002340050349

[25] van Geel, B.M., Bezman, L., Loes, D.J., Moser, H.W. and Raymond, G.V. (2001) Evolution of Phenotypes in Adult Male Patients with X-Linked Adrenoleukodystrophy. Annals of Neurology, 49, 186-194. http://dx.doi.org/10.1002/1531-8249(20010201)49:2<186::AID-ANA38>3.0.CO;2-R 